\title{
BANACH SPACES IN WHICH EVERY COMPACT LIES INSIDE THE RANGE OF A VECTOR MEASURE
}

\author{
C. PIÑEIRO AND L. RODRÍGUEZ-PIAZZA
}

(Communicated by Andrew M. Bruckner)

\begin{abstract}
We prove that the compact subsets of a Banach space $X$ lie inside ranges of $X$-valued measures if and only if $X^{*}$ can be embedded in an $L^{1}$ space. In these spaces we prove that every compact is, in fact, a subset of a compact range. We also prove that if every compact of $X$ is a subset of the range of an $X$-valued measure of bounded variation, then $X$ is finite dimensional. Thus we answer a question by R. Anantharaman and J. Diestel.
\end{abstract}

\section{INTRODUCTION}

In [AD] the authors proved that some Banach spaces have the property that their compact sets are subsets of ranges of vector measures. They asked for a characterization of these Banach spaces. We prove that they are those Banach spaces whose dual can be embedded in an $L^{1}$ space. We also prove that in these spaces every compact lies in the range of measure with compact range. These two assertions are contained in Theorem 3.6, which is our main result.

Another natural question arises if we require these measures to have bounded variation; that is, which Banach spaces have the property that every compact subset lies in the range of a measure of bounded variation? We answer this question in Theorem 2.1, only finite-dimensional Banach spaces have this property. We use different approaches to answer these two questions, but they are interchangeable (see Remark 3.7).

In $\S 1$ we introduce the notations and recall some results about ranges of vector measures. Section 2 is devoted to proving Theorem 2.1. This is done by studying the finite-dimensional quotient spaces of the Banach space having the property required in the second question.

In $\S 3$ we give the answer to the question by Anantharaman and Diestel. First let us note that this question remains equivalent if we substitute compact sets by norm null sequences. That is the reason why we introduce the spaces $R(X)$ and $R_{c}(X)$ of sequences in a Banach space $X$ lying inside the range of an $X$-valued measure or of an $X$-valued measure with compact range. The study of these spaces allows us to prove our main result. We complete this section

Received by the editors August 21, 1990.

1980 Mathematics Subject Classification (1985 Revision). Primary 28B05, 46G10; Secondary 46B20, 47D30.

Key words and phrases. Vector measures, range, Banach spaces, compact sets, subspaces of $L^{1}$. 
with an example of a compact subset of the range of a measure that is not a subset of any compact range.

\section{NOTATIONS AND PRELIMINARIES}

We use the classical notation in Banach space theory. We consider all Banach space over real numbers. If $X$ is a Banach space, $X^{*}$ denotes its dual space, and $B_{X}$ its closed unit ball. For a subset $K$ of $X, \overline{c o}(K)$ is the closed convex hull of $K$. As usual $c_{0}(X)\left(l_{1}(X)\right)$ stands for the Banach space of norm null sequences (absolutely convergent series) in $X$; and $l_{p}^{n}$ is $\mathbb{R}^{n}$ equipped with the norm $\|\cdot\|_{p}, 1 \leq p \leq \infty$. In this case $B_{p}^{n}$ is its closed unit ball $\left(B_{p}^{n}=B_{l_{p}^{n}}\right)$.

We refer to $[\mathrm{P}]$ or $[\mathrm{J}]$ for the definition of the nuclear and $p$-summing norm $(1 \leq p<\infty)$ of an operator $T$, denoted respectively by $n(T)$ and $\pi_{p}(T)$. If $X$ and $Y$ are Banach spaces, $N(X, Y)\left(\Pi_{p}(X, Y)\right)$ will be the space of nuclear ( $p$-summing) operators from $X$ into $Y$.

We consider only countably additive measures defined on $\sigma$-algebras. If $\Sigma$ is a $\sigma$-algebra of subsets of a set $\Omega, X$ is a Banach space, and $\mu: \Sigma \rightarrow X$ is such a measure, we denote by $|\mu|$ the variation of $\mu$, which is an extended positive measure; by $\operatorname{tv}(\mu)$ its total variation, that is, $\operatorname{tv}(\mu)=|\mu|(\Omega)$, and by $\|\mu\|$ its (total) semivariation:

$$
\|\mu\|=\sup \left\{\left|x^{*} \circ \mu\right|(\Omega): x^{*} \in B_{X^{*}}\right\} .
$$

If $\operatorname{tv}(\mu)<+\infty$, we say that $\mu$ has bounded variation. The range of $\mu$ is denoted by $\operatorname{rg} \mu$, that is, $\operatorname{rg} \mu=\{\mu(A): A \in \Sigma\}$.

We need several known results providing us the existence of plenty of ranges of measures. We summarize them in the next three propositions.

Proposition 1.1. If $X$ is a Banach space and $\mu$ is an $X$-valued measure, then there exists an $X$-valued measure $\nu$ such that $\overline{\operatorname{co}}(\operatorname{rg} \mu)=\operatorname{rg} \nu$. In this case, we have $\|\mu\|=\|\nu\|$ and $\operatorname{tv}(\mu)=\operatorname{tv}(\nu)$.

This proposition is very useful in order to reduce the study of compact sets lying inside ranges of measures to the case of null sequences; for the proof of the existence of $\nu$ see [DU, p. 279] or [KK, p. 128]. For the last assertion see [R].

The next proposition can easily be proved using a direct sum of vector measures [KK, p. 35].

Proposition 1.2. Let $X$ be a Banach space and $\left(\mu_{n}\right)$ a sequence of $X$-valued measures. If $\sum\left\|\mu_{n}\right\|<+\infty$ (resp. $\left.\sum \operatorname{tv}\left(\mu_{n}\right)<+\infty\right)$, then there exists an $X$-valued measure $\mu$ (resp. of bounded variation) such that

(a) $\operatorname{rg} \mu=\sum \operatorname{rg} \mu_{n}=\left\{\sum_{n=1}^{\infty} x_{n}: x_{n} \in \operatorname{rg} \mu_{n}\right\}$.

(b) $\|\mu\| \leq \sum\left\|\mu_{n}\right\|, \operatorname{tv}(\mu)=\sum \operatorname{tv}\left(\mu_{n}\right)$.

In particular, $\operatorname{rg} \mu_{n} \subset \operatorname{rg} \mu$ for every $n$.

If $K$ is a compact Hausdorff space and $T: C(K) \rightarrow X$ is a weakly compact operator, then there is an $X$-valued measure $\nu$ defined on the Borel subsets of $K$ representing the operator $T$ [DU]. A consequence of the regularity of $\nu$ is that

$$
\overline{\mathrm{co}}(\mathrm{rg} \nu)=\overline{\{T(f): f \in C(K), 0 \leq f \leq 1\}},
$$

and the closure of $T\left(B_{C(K)}\right)$ is $\overline{\mathrm{co}}(\mathrm{rg} \nu-\mathrm{rg} \nu)$. Then, by the last two propositions and the properties of the representing measure, we have 
Proposition 1.3. If $T: C(K) \rightarrow X$ is a weakly compact operator, then there exists an $X$-valued measure $\mu$ such that

$$
\overline{T\left(B_{C(K)}\right)}=\operatorname{rg} \mu \text { and }\|\mu\|=2\|T\| .
$$

If $T$ is 1-summing, then $\mu$ has bounded variation and $\operatorname{tv}(\mu)=2 \pi_{1}(T)$.

Finally, we introduce a very special kind of ranges, which we call a countable sum of segments. If $\sum x_{n}$ is an unconditionally convergent series in a Banach $X$, it is known that for any bounded sequence of real numbers $\left(\alpha_{n}\right)$ the series $\sum \alpha_{n} x_{n}$ is also convergent, so we can define the sum of the segments $\left[-x_{n}, x_{n}\right]$ as

$$
\sum_{n=1}^{\infty}\left[-x_{n}, x_{n}\right]=\left\{\sum_{n=1}^{\infty} \alpha_{n} x_{n}:\left(\alpha_{n}\right) \in l_{\infty},\left\|\left(\alpha_{n}\right)\right\|_{\infty} \leq 1\right\} .
$$

This is a compact convex subset of $X$. The operator $T: l_{\infty} \rightarrow X$ defined by $T\left(\alpha_{n}\right)=\sum \alpha_{n} x_{n}$ is weak*-weak continuous (here weak* means that we consider $l_{\infty}$ as the dual of $\left.l_{1}\right) . T$ is a compact operator and we see

$$
\sum_{n=1}^{\infty}\left[-x_{n}, x_{n}\right]=T\left(B_{l_{\infty}}\right)=\overline{T\left(B_{c_{0}}\right)} .
$$

Then a countable sum of segments is the range of a measure $\left(l_{\infty}\right.$ is a $C(K)$ space) with compact range. The next proposition shows that a subset of a Banach space lies inside a countable sum of segments if and only if it lies inside the range of a measure with (relatively) compact range. We will use this fact in $\S 3$.

Proposition 1.4. Let $X$ be a Banach space and $\mu$ an $X$-valued measure with relatively compact range. Then there exists an unconditionally convergent series $\sum x_{n}$ in $X$ such that

$$
\operatorname{rg} \mu \subset \sum_{n=1}^{\infty}\left[-x_{n}, x_{n}\right]
$$

Proof. There exist a probability measure $\lambda$ and a compact operator $T: L^{\infty}(\lambda) \rightarrow$ $X$ such that $\operatorname{rg} \mu \subset T\left(B_{L^{\infty}}\right)$ and $T$ is weak*-weak continuous (i.e.: $T^{*}\left(X^{*}\right) \subset$ $\left.L^{1}(\lambda)\right)$ [DU, p. 263]. First we prove the following:

Claim. In the above conditions, given $\varepsilon>0$, there exist $m \in \mathbb{N}$ and two operators $R: l_{\infty}^{m} \rightarrow X$ and $S: L^{\infty}(\lambda) \rightarrow X$ satisfying

(a) $T\left(B_{L^{\infty}(\lambda)}\right) \subset R\left(B_{\infty}^{m}\right)+S\left(B_{L^{\infty}(\lambda)}\right)$.

(b) $S$ is weak*-weak continuous and compact.

(c) $\|R\| \leq\|T\|$ and $\|S\| \leq \varepsilon$.

$T^{*}$ is a compact operator, so there is a norm one projection $P$ in $L^{1}(\lambda)$, which is a conditional expectation over a finite $\sigma$-algebra, such that

$$
\|y-P y\| \leq \varepsilon \text { for all } y \in T^{*}\left(B_{X^{*}}\right) .
$$

That is, $\left\|T^{*}-P T^{*}\right\| \leq \varepsilon$. Take $S=\left(T^{*}-P T^{*}\right)^{*}=T-T P^{*}$. Since $T$ is compact and weak*-weak continuous and $P^{*}$ is weak*-weak* continuous, we have that $S$ satisfies (b) and $\|S\| \leq \varepsilon . P^{*}$ is the same conditional expectation (taken in $L^{\infty}(\lambda)$ ) and its range is a subspace $Y$ of finite dimension $m$ isometric to $l_{\infty}^{m}$. Composing this isometry with $\left.T\right|_{Y}$ we get an operator $R: l_{\infty}^{m} \rightarrow X$ such 
that $\|R\| \leq\|T\|$. Since $R\left(B_{\infty}^{m}\right)=T\left(B_{Y}\right)$ and $P^{*}$ has norm one, the equality $T=S+T P^{*}$ implies that (a) is satisfied. This proves the claim.

Let us write $T_{0}=T$ and apply the claim for $\varepsilon=\varepsilon_{0}=\|T\| / 2$; then we obtain $m_{1} \in \mathbb{N}$ and operators

$$
R_{1}: l_{\infty}^{m_{1}} \rightarrow X \text { and } S: L^{\infty}(\lambda) \rightarrow X
$$

satisfying (a), (b), and (c). Put $T_{1}=S$ and apply again the claim to $T_{1}$ for $\varepsilon=\varepsilon_{1}=\varepsilon_{0} 2^{-1}$. An inductive procedure gives us a sequence $\left(m_{n}\right)$ of natural numbers and two operator sequences $R_{n}: l_{\infty}^{m_{n}} \rightarrow X, T_{n}: L^{\infty}(\lambda) \rightarrow X$ satisfying

(i) $\left\|R_{n}\right\| \leq\left\|T_{n-1}\right\|, n=1,2, \ldots$;

(ii) $\left\|T_{n}\right\| \leq\|T\| 2^{-n}, n=0,1, \ldots$;

(iii) $T_{n}\left(B_{L^{\infty}}\right) \subset R_{n+1}\left(B_{\infty}^{m_{n}}\right)+T_{n+1}\left(B_{L^{\infty}}\right), n=0,1, \ldots$

From (i) and (ii) we obtain

$$
\sum_{n=1}^{\infty}\left\|R_{n}\right\| \leq 2\|T\|
$$

And by (iii) and (ii) we have for every $n$

$$
T\left(B_{L^{\infty}}\right) \subset \sum_{k=1}^{n} R_{k}\left(B_{\infty}^{m_{k}}\right)+T_{n}\left(B_{L^{\infty}}\right) \subset \sum_{k=1}^{n} R_{k}\left(B_{\infty}^{m_{k}}\right)+\|T\| 2^{-n} B_{X} .
$$

We can consider the sequence $\left(l_{\infty}^{m_{n}}\right)$ placed pairwise disjointly in $c_{0}$ (take a sequence $\left(A_{n}\right)$ of disjoint subsets of $\mathbb{N}$ with $\left|A_{n}\right|=m_{n}$ and consider $l_{\infty}^{m_{n}}$ as the subspace generated by $\left\{e_{j}: j \in A_{n}\right\}$, where $\left\{e_{j}: j \in \mathbb{N}\right\}$ is the canonical basis of $c_{0}$ ). Let $P_{n}: c_{0} \rightarrow l_{\infty}^{m_{n}}$ be the natural projection and take $U_{n}=R_{n} P_{n}$. By (2) we can define a compact operator $U: c_{0} \rightarrow X$ as $U=\sum_{n=1}^{\infty} U_{n}$. Since the $l_{\infty}^{m_{n}}$ are placed disjointly, for every $n$ and every $z$ in $\sum_{k=1}^{n} R_{k}\left(B_{\infty}^{m_{k}}\right)$, there exists $y \in B_{c_{0}}$ such that $U y=z$. Then by (3) we obtain

$$
\operatorname{rg} \mu \subset T\left(B_{L^{\infty}}\right) \subset \overline{U\left(B_{c_{0}}\right)} .
$$

Taking $x_{n}=U e_{n}$ the proposition follows.

\section{COMPACT SETS IN RANGES OF MEASURES OF BOUNDED VARIATION}

We devote this section to prove that every compact set of a given Banach space $X$ lies inside the range of an $X$-valued measure of bounded variation if and only if $X$ is finite dimensional. Since the "if" part is obvious, it suffices to prove the "only if" part, which is Theorem 2.1. In the proof of this theorem it is given (Lemma 2.2) a lower bound of the total variation of a measure valued in a finite-dimensional Banach space and containing the unit ball in its range.

Theorem 2.1. Let $X$ be a Banach space. If every compact subset of $X$ lies inside the range of an $X$-valued measure of bounded variation, then $X$ is finite dimensional.

Proof. First let us establish the following:

Claim. Under the hypothesis of the theorem, there exists a constant $c>0$ such that every finite subset of $B_{X}$ lies inside the range of a measure $\mu$ with total variation $\operatorname{tv}(\mu) \leq c$. 
If the claim were false, there would exist a sequence $\left(F_{n}\right)$ of finite subsets of $B_{X}$ such that for every $n$ the condition $F_{n} \subset \operatorname{rg} \mu$ would imply $\operatorname{tv}(\mu) \geq n^{2}$. Being the set $K=\bigcup_{n=1}^{\infty} \frac{1}{n} F_{n}$ relatively compact (it is a null sequence); there is a measure of bounded variation $\mu$ with $K \subset \operatorname{rg} \mu$. Thus $F_{n} \subset \operatorname{rg} n \mu$ and this would yield $\operatorname{tv}(\mu) \geq n$ for every $n$, a contradiction with the fact that $\mu$ has bounded variation.

In order to finish the proof we need the following lemma.

Lemma 2.2. Let $E$ be a finite-dimensional Banach space. If $\delta \in(0,1)$ and $\mu:(\Sigma, \Omega) \rightarrow E$ is a measure such that for every $x \in S_{E}$ there exists $A \in \Sigma$ with $\|\mu(A)-x\| \leq \delta$, then

$$
\operatorname{tv}(\mu) \geq(1-\delta) \pi_{1}\left(I_{E^{*}}\right) \geq(1-\delta) \sqrt{\operatorname{dim}(E)} .
$$

End of the proof of Theorem 2.1. Let $X$ be an infinite-dimensional Banach space, and let $Y$ be a closed subspace of finite codimension $n$. We can take a finite subset $H$ of the open unit ball of $E=X / Y$ such that $d_{E}(x, H) \leq \frac{1}{2}$ for every $x \in S_{E}$. If $Q: X \rightarrow E$ is the quotient map, we can find a finite subset $J$ of $B_{X}$ such that $Q(J)=H$. If $\mu$ is an $X$-valued measure with $J \subset \operatorname{rg} \mu$, then $Q \circ \mu$ is an $E$-valued measure with $H \subset \operatorname{rg}(Q \circ \mu)$. From Lemma 2.2 it follows that $\operatorname{tv}(\mu) \geq \operatorname{tv}(Q \circ \mu) \geq \frac{1}{2} \sqrt{n}$. As $n$ was arbitrary, $X$ cannot satisfy the claim and the thoerem follows.

Proof of Lemma 2.2. The hypothesis of the lemma imply that for every $x^{*} \in E^{*}$ we have

$$
\sup \left\{\left\langle x^{*}, \mu(A)\right\rangle: A \in \Sigma\right\} \geq(1-\delta)\left\|x^{*}\right\| .
$$

Let $f$ be the Radon-Nikodym derivative of $\mu$ with respect to its variation $|\mu|$. We know that $\|f(\omega)\|=1 \quad|\mu|$-almost everywhere, so

$$
\left\|x^{*} \circ f\right\|_{L^{\infty}(|\mu|)} \leq\left\|x^{*}\right\| \text { for all } x^{*} \in E^{*} \text {. }
$$

By (4) we also have

$$
(1-\delta)\left\|x^{*}\right\| \leq \sup \left\{\int_{A}\left(x^{*} \circ f\right) d|\mu|: A \in \Sigma\right\} \leq\left\|x^{*} \circ f\right\|_{L^{1}(|\mu|)} .
$$

Let $F$ be the space $F=\left\{x^{*} \circ f: x^{*} \in E^{*}\right\}$. Let us write $F_{\infty}$ and $F_{1}$ when we consider in $F$ the norms in $L^{\infty}(|\mu|)$ and in $L^{1}(|\mu|)$ respectively. Inequalities (5) and (6) allow us to define two operators $T: E^{*} \rightarrow F_{\infty}$ and $S: F_{1} \rightarrow E^{*}$ as $T x^{*}=x^{*} \circ f$ and $S\left(x^{*} \circ f\right)=x^{*}$ for all $x^{*} \in E^{*}$. We have $\|T\| \leq 1$ and $\|S\| \leq(1-\delta)^{-1}$. Now we consider the identity map $R$ from $F_{\infty}$ into $F_{1}$. We have $I_{E^{*}}=S \circ R \circ T$. From the easy part of the "Pietsch Factorization Theorem" for 1-summing operators, we obtain $\pi_{1}(R) \leq \operatorname{tv}(\mu)$ and the first inequality follows from the ideal property of $\Pi_{1}$. The second inequality is classical: $\pi_{1}\left(I_{E^{*}}\right) \geq \pi_{2}\left(E_{E^{*}}\right)=\sqrt{\operatorname{dim}(E)}$ (see, for instance, [P, Chapter 1]).

When we use Lemma 2.2 to prove the Theorem we apply it to the finitedimensional quotients of $X$. Since we allow the measures to be $X$-valued, we cannot apply it to a finite-dimensional subspace $E$ of $X$. Moreover, as we show in the next example, there are measures of bounded variation whose ranges contain the unit ball of infinite-dimensional subspaces. The key is that there are quotient maps that are 1-summing, in contradiction to the fact that an 
embedding is 1-summing only if it has a finite rank, whose quantitative version we have used.

Example 2.3. An $l_{\infty}$-valued measure of bounded variation whose range contains the unit ball of an infinite-dimensional subspace.

Let $Y$ be a subspace of $l_{\infty}$ isometric to $l_{2}$, and consider a quotient map $Q: l_{1} \rightarrow Y . Q$ is a bounded operator such that $B_{Y} \subset \overline{Q\left(B_{l_{1}}\right)}$. Since $Q$ is an operator from an $L^{1}$-space into a Hilbert space, it follows that $Q$ is 1 summing thanks to Grothendieck's Theorem [P, Theorem 5.12]. By Pietsch's Factorization Theorem [P, Theorem 1.3] there exist a compact space $K$, a Radon probability $\lambda$ on $K$, a closed subspace $F$ of $C(K)$, and continuous operators $R: l_{1} \rightarrow F$ and $S: \bar{F}^{L^{1}(\lambda)} \rightarrow Y$ such that

$$
\|R\|=1, \quad\|S\|=\pi_{1}(Q), \quad \text { and } Q=\left.S \circ j\right|_{F} \circ R,
$$

where $j: C(K) \rightarrow L^{1}(\lambda)$ is the natural inclusion. This implies $\left.B_{Y} \subset S \circ j\right|_{F}\left(B_{F}\right)$. Thanks to the "extension property" of $l_{\infty}$ we can extend $S$ to $L^{1}(\lambda)$. That is, there exists $\widetilde{S}: L^{1}(\lambda) \rightarrow l_{\infty}$ such that $\left.\widetilde{S}\right|_{F_{1}}=S$, where $F_{1}=\bar{F}^{L^{1}(\lambda)}$. The composition $T=\widetilde{S} \circ j$ provides us a 1 -summing operator ( $j$ is 1-summing) such that $B_{Y} \subset \overline{T\left(B_{C(K)}\right)}$. By Proposition 1.3 there is an $l_{\infty}$-valued measure of bounded variation $\mu$ such that $B_{Y} \subset \operatorname{rg} \mu$.

\section{The SPACes $R(X)$ AND $R_{c}(X)$. The MAIN RESUlt}

Since every norm compact subset of a Banach space is contained in the closed convex hull of a norm null sequence [D, p. 3], it follows from Proposition 1.1 that the two following statements are equivalent:

(i) Every compact subset of $X$ lies inside the range of an $X$-valued measure.

(ii) Every norm null sequence in $X$ lies inside the range of an $X$-valued measure.

In this section it is proved that $X$ satisfies the statements (i), (ii) if and only if $X^{*}$ is isomorphic to a subspace of an $L^{1}$ space.

We denote by $R(X)$ the vector space of all sequences $\left(x_{n}\right)$ in $X$ so that there exists an $X$-valued measure $\mu$ satisfying

$$
\left\{x_{n}: n \in \mathbf{N}\right\} \subset \operatorname{rg} \mu \text {. }
$$

If $\left(x_{n}\right)$ belongs to $R(X)$, we put $\left\|\left(x_{n}\right)\right\|_{r}=\inf \|\mu\|$, where the infimum is taken over all vector measures $\mu$ admissible in (7). Obviously, we have

$$
\left\|\left(x_{n}\right)\right\|_{\infty} \leq\left\|\left(x_{n}\right)\right\|_{r} \text { for all }\left(x_{n}\right) \in R(X)
$$

Thus statement (i) is equivalent to the next one: $c_{0}(X)$ is contained in $R(X)$.

Proposition 3.1. (a) $\left(R(X),\|\cdot\|_{r}\right)$ is a Banach space.

(b) $c_{0}(X)$ is contained in $R(X)$ if and only if there exists a constant $c>0$ such that $\left\|\left(x_{n}\right)\right\|_{r} \leq c\left\|\left(x_{n}\right)\right\|_{\infty}$ for all sequences $\left(x_{n}\right)$ that contain only finitely many nonzero terms.

Proof. We omit the straightforward verifications of statement (a) and make only a remark on the completeness assertion. To this end, it suffices to prove that any absolutely summable series in $R(X)$ is convergent, and this follows easily using Proposition 1.2. 
The statement (b) is an obvious consequence of inequality (8), the Open Mapping Theorem, and the density in $c_{0}(X)$ of the sequences $\left(x_{n}\right)$ with only finitely many nonzero terms.

Remark 3.2. From Proposition 3.1(b) it follows that $c_{0}(X)$ is contained in $R(X)$ if and only if there exists a constant $c>0$ such that the following is true: for every finite subset $H$ of $B_{X}$ there is an $X$-valued measure $\mu$ so that $H \subset \operatorname{rg} \mu$ and $\|\mu\| \leq c$.

The next lemma contains some properties of Banach spaces $X$ satisfying the condition $c_{0}(X) \subset R(X)$.

Lemma 3.3. (a) $c_{0}\left(L^{\infty}(\lambda)\right) \subset R\left(L^{\infty}(\lambda)\right)$ for every extended positive measure $\lambda$.

(b) If $c_{0}(X) \subset R(X)$, then $c_{0}(Z) \subset R(Z)$ for every quotient $Z$ of $X$.

(c) If $c_{0}\left(X^{* *}\right) \subset R\left(X^{* *}\right)$, then $c_{0}(X) \subset R(X)$.

Proof. (a) Let $H \subset B_{X}$ be a finite set. Given $\varepsilon>0$, there exist a finitedimensional subspace $Y$ of $X$ and $T: l_{\infty}^{n} \rightarrow Y$ so that $H \subset Y,\left\|T^{-1}\right\|=1$, and $\|T\| \leq 1+\varepsilon$. As $H \subset T\left(B_{\infty}^{n}\right)$, by Proposition 1.3 there exists a $Y$-valued measure $\mu$ so that

$$
H \subset \operatorname{rg} \mu \text { and }\|\mu\| \leq 2(1+\varepsilon) .
$$

(b) Let $Y$ be a closed subspace of $X$. By Remark 3.2, there is a constant $c>0$ such that every finite subset of $B_{X}$ lies inside the range of an $X$-valued measure $\mu$ with $\|\mu\| \leq c$. Given $\varepsilon>0$, if $H=\left\{\bar{x}_{1}, \ldots, \bar{x}_{n}\right\}$ is a finite subset of the closed unit ball of $X / Y$, we can choose $x_{i} \in \bar{x}_{i}$ so that $\left\|x_{i}\right\| \leq 1+\varepsilon$ for all $i \leq n$. Let $\mu$ be an $X$-valued measure such that

$$
\left\{x_{i}\right\}_{i=1}^{n} \subset \operatorname{rg} \mu \text { and }\|\mu\| \leq(1+\varepsilon) c .
$$

If $\varphi: X \rightarrow X / Y$ is the canonical surjection, $\mu_{1}=\varphi \circ \mu$ is an $X / Y$-valued measure for which we have

$$
H \subset \operatorname{rg} \mu_{1} \text { and }\left\|\mu_{1}\right\| \leq(1+\varepsilon) c .
$$

(c) Again by the above remark there exists a constant $c>0$ so that every finite subset $H \subset B_{X}$ *. lies inside the range of an $X^{* *}$-valued measure $\mu$ for which

$$
H \subset \operatorname{rg} \mu \text { and }\|\mu\| \leq c .
$$

So, if $\left\{x_{i}\right\}_{i=1}^{n}$ is a finite subset of $B_{X}$, there is an $X^{* *}$-valued measure $\mu$ such that $\left\{x_{i}\right\}_{i=1}^{n} \subset \operatorname{rg} \mu$ and $\|\mu\| \leq c$. Choose $A_{i}$ so that $x_{i}=\mu\left(A_{i}\right)$, and consider the $\sigma$-field $\Sigma_{0}$ generated by $\left\{A_{1}, \ldots, A_{n}\right\}$ on $\Omega$. Let $\tilde{\mu}$ be the restriction of $\mu$ to $\Sigma_{0}$, and $Y$ be the finite-dimensional linear span of $\operatorname{rg} \tilde{\mu}$; by the "principle of Local Reflexivity" ([De] or [P, p. 6]), given $\varepsilon>0$, there exists a one-to-one operator $T: Y \rightarrow X$ with $T x=x$ for all $x \in X \cap Y$, and $\|T\| \leq 1+\varepsilon$. Thus $\mu_{1}=T \circ \tilde{\mu}$ is an $X$-valued measure for which we have $\left\{x_{i}\right\}_{i=1}^{n} \subset \operatorname{rg} \mu_{1}$ and $\left\|\mu_{1}\right\| \leq(1+\varepsilon) c$. The lemma follows from Remark 3.2.

Next we are going to consider sequences in $X$ that lie inside the range of a vector measure with relatively compact range. We denote by $R_{c}(X)$ the vector space of all such sequences $\left(x_{n}\right)$ in $X$. By Proposition 1.4, if $\left(x_{n}\right)$ belongs to $R_{c}(X)$, there exists an unconditionally convergent series $\sum y_{k}$ satisfying $x_{n} \in \sum\left[-y_{k}, y_{k}\right]$ for all $n$. Recall that $\sum\left[-y_{k}, y_{k}\right]$ is the range of a vector measure $\mu$ for which

$$
\|\mu\| \leq 2 \sup \left\{\sum_{k=1}^{\infty}\left|\left\langle y_{k}, x^{*}\right\rangle\right|: x^{*} \in B_{X} \cdot\right\} .
$$


If $\left(x_{n}\right)$ belongs to $R_{c}(X)$, we set

$$
\left\|\left(x_{n}\right)\right\|_{r c}=\inf \sup \left\{\sum_{k=1}^{\infty}\left|\left\langle y_{k}, x^{*}\right\rangle\right|: x^{*} \in B_{X^{*}}\right\},
$$

the infimum being taken over all u.c. series $\sum y_{k}$ such that $\left\{x_{n}\right\}$ is contained in $\sum\left[-y_{k}, y_{k}\right]$. Obviously, we have

$$
\left\|\left(x_{n}\right)\right\|_{\infty} \leq\left\|\left(x_{n}\right)\right\|_{r} \leq 2\left\|\left(x_{n}\right)\right\|_{r c} \text { for all }\left(x_{n}\right) \in R_{c}(X) .
$$

Proposition 3.4. (a) $\left(R_{c}(X),\|\cdot\|_{r c}\right)$ is a Banach space.

(b) $c_{0}(X)$ is contained in $R_{c}(X)$ if and only if there is a constant $c>0$ such that $\left\|\left(x_{n}\right)\right\|_{r c} \leq c\left\|\left(x_{n}\right)\right\|_{\infty}$ for all sequences $\left(x_{n}\right)$ that contain only finitely many nonzero terms.

(c) If $\left(x_{n}^{*}\right)$ is a sequence in $X^{*}$ for which the operator $T: x \in X \rightarrow\left(\left\langle x, x_{n}^{*}\right\rangle\right) \in$ $l_{1}$ is 1-summing, then the linear form $\varphi$ defined by

$$
\varphi:\left(x_{n}\right) \in R_{c}(X) \rightarrow \sum_{n=1}^{\infty}\left\langle x_{n}, x_{n}^{*}\right\rangle \in \mathbb{R}
$$

is well defined and continuous.

Proof. Again the completeness of $R_{c}(X)$ is proved showing that any absolutely summable series in $R_{c}(X)$ is convergent. Then (a) and (b) follow easily as in Proposition 3.1.

(c) Let $\left(x_{n}\right)$ be a sequence belonging to $R_{c}(X)$. Choose an u.c. series $\sum y_{k}$ so that

$$
\left\{x_{n}: n \in \mathbf{N}\right\} \subset\left\{\sum_{k=1}^{\infty} \alpha_{k} y_{k}:\left|\alpha_{k}\right| \leq 1\right\} .
$$

Then we have

$$
\begin{aligned}
\sum_{n=1}^{\infty}\left|\left\langle x_{n}, x_{n}^{*}\right\rangle\right| & \leq \sum_{n=1}^{\infty} \sum_{k=1}^{\infty}\left|\left\langle y_{k}, x_{n}^{*}\right\rangle\right|=\sum_{k=1}^{\infty}\left\|\left(\left\langle y_{k}, x_{n}^{*}\right\rangle\right)_{n}\right\|_{1} \\
& \leq \pi_{1}(T) \sup \left\{\sum_{k=1}^{\infty}\left|\left\langle y_{k}, x^{*}\right\rangle\right|: x^{*} \in B_{X^{*}}\right\} .
\end{aligned}
$$

Hence $\varphi$ is well defined and continuous since

$$
\left.\left|\left\langle\varphi,\left(x_{n}\right)\right\rangle\right| \leq \pi_{1}(T) \|\left(x_{n}\right)\right)_{r c} .
$$

Remark 3.5. From Proposition 3.4(b) it follows that $c_{0}(X)$ is contained in $R_{c}(X)$ if and only if there exists a constant $c>0$ such that the following is true: for every finite subset $H$ of $B_{X}$, there is a finite subset $\left\{y_{1}, \ldots, y_{n}\right\}$ of $X$ so that

$$
H \subset\left\{\sum_{i=1}^{n} \alpha_{i} y_{i}:\left|\alpha_{i}\right| \leq 1\right\} \text { and } \sup \left\{\sum_{i=1}^{n}\left|\left\langle y_{i}, x^{*}\right\rangle\right|: x^{*} \in B_{X^{*}}\right\} \leq c .
$$

It can be proved that the linear form defined in Proposition 3.4(c) is also continuous on $R(X)$. Since we need only the continuity on $R_{c}(X)$, we do not include the larger proof of this fact. Now we are ready for the main theorem. 
Theorem 3.6. Let $X$ be a Banach space. The following statements are equivalent:

(a) Every norm compact subset of $X$ lies inside the range of an $X$-valued measure.

(b) $c_{0}(X) \subset R(X)$.

(c) $c_{0}(X) \subset R_{c}(X)$.

(d) $\Pi_{1}\left(X, l_{1}\right)=N\left(X, l_{1}\right)$.

(e) $X^{*}$ is isomorphic to a subspace of an $L^{1}$ space.

Proof. We already know that (a) and (b) are equivalent.

(b) $\Rightarrow$ (c). By Remark 3.2, there is a constant $c>0$ such that, for every finite subset $\left\{x_{n}, \ldots, x_{n}\right\}$ of $B_{X}$, there exists an $X$-valued measure $\mu$ satisfying

$$
\left\{x_{i}\right\}_{i=1}^{n} \subset \operatorname{rg} \mu \text { and }\|\mu\| \leq c .
$$

Choose $A_{i}$ such that $x_{i}=\mu\left(A_{i}\right)$ and consider the $\sigma$-field $\Sigma_{0}$ generated by $\left\{A_{1}, \ldots, A_{n}\right\}$ on $\Omega$. If $E_{1}, \ldots, E_{m}$ are the atoms of $\Sigma_{0}$, we put $y_{j}=\mu\left(E_{j}\right)$ for $j \leq m$. Let us note that

$$
\left\{x_{i}\right\}_{i=1}^{n} \subset\left\{\sum_{j=1}^{m} \alpha_{j} y_{j}:\left|\alpha_{j}\right| \leq 1\right\}
$$

and

$$
\sup \left\{\sum_{j=1}^{m}\left|\left\langle y_{j}, x^{*}\right\rangle\right|: x^{*} \in B_{X^{*}}\right\} \leq\|\mu\| \leq c .
$$

Hence (c) follows from Remark 3.5.

$(c) \Rightarrow(d)$. From (10) and the Open Mapping Theorem it follows that there is a constant $c>0$ so that

$$
\left\|\left(x_{n}\right)\right\|_{r c} \leq c\left\|\left(x_{n}\right)\right\|_{\infty} \text { for all }\left(x_{n}\right) \in c_{0}(X) .
$$

If $T: X \rightarrow l_{1}$ is a 1-summing operator, then $T x=\left(\left\langle x, x_{n}^{*}\right\rangle\right)$ for a suitable sequence $\left(x_{n}^{*}\right)$ in $X^{*}$, and by Proposition 3.4(c), the linear form

$$
\varphi:\left(x_{n}\right) \in R_{c}(X) \rightarrow \sum_{n=1}^{\infty}\left\langle x_{n}, x_{n}^{*}\right\rangle \in \mathbb{R}
$$

is continuous. From (12) it follows that the restriction map of $\varphi$ to $c_{0}(X)$ is also continuous; so $\varphi$ belongs to $l_{1}\left(X^{*}\right)$, the dual space of $c_{0}(X)$. Then $\sum\left\|x_{n}^{*}\right\|<+\infty$ and this proves that $T$ is nuclear.

(d) $\Rightarrow$ (e). J. Lindenstrauss and A. Pełczynski [LP] proved that a Banach space $X$ is isomorphic to a subspace of an $L^{1}$ space if and only if there exists a constant $c>0$ such that the following is true: If $H$ and $G$ are finite subsets of $X$ so that

$$
\sum_{x \in H}\left|\left\langle x, x^{*}\right\rangle\right| \leq \sum_{y \in G}\left|\left\langle y, x^{*}\right\rangle\right| \text { for all } x^{*} \in X^{*},
$$

then $\sum_{x \in H}\|x\| \leq c \sum_{y \in G}\|y\|$.

We also recall that the Banach spaces $N\left(X, l_{1}\right)$ and $l_{1}\left(X^{*}\right)$ are isometric [J, 1.15]. Assume that (d) holds; then there exists a constant $c>0$ such that

$$
\mathrm{n}(T) \leq c \pi_{1}(T) \text { for all } T \in N\left(X, l_{1}\right) .
$$


If $\left\{x_{i}^{*}\right\}_{i=1}^{n}$ and $\left\{y_{j}^{*}\right\}_{j=1}^{m}$ are finite subsets of $X^{*}$ so that

$$
\sum_{i=1}^{n}\left|\left\langle x_{i}^{*}, x^{* *}\right\rangle\right| \leq \sum_{j=1}^{m}\left|\left\langle y_{j}^{*}, x^{* *}\right\rangle\right| \text { for all } x^{* *} \in X^{* *},
$$

we can define two linear operators $T$ and $S$ from $X$ into $l_{1}$ by

$$
T x=\sum_{i=1}^{n}\left\langle x, x_{i}^{*}\right\rangle e_{i} \text { and } S x=\sum_{j=1}^{m}\left\langle x, y_{j}^{*}\right\rangle e_{j}
$$

for all $x \in X$, where $\left\{e_{i}: i \in \mathbb{N}\right\}$ is the unit basis of $l_{1}$. From (14) it follows that $\pi_{1}(T) \leq \pi_{1}(S)$. This and (13) yields

$$
\mathrm{n}(T) \leq c \pi_{1}(T) \leq c \pi_{1}(S) \leq c \mathrm{n}(S) ;
$$

but $\mathrm{n}(T)=\sum_{i=1}^{n}\left\|x_{i}^{*}\right\|$ and $\mathrm{n}(S)=\sum_{j=1}^{m}\left\|y_{j}^{*}\right\|$, so the implication follows by the Lindenstrauss-Petczynski characterization.

(e) $\Rightarrow\left(\right.$ b). If $X^{*}$ is isomorphic to a subspace of an $L^{1}$ space, then $X^{* *}$ is isomorphic to a quotient of an $L^{\infty}$ space. Lemma 3.3 assures us that $c_{0}(X) \subset$ $R(X)$.

Remark 3.7. In order to prove Theorem 3.6 we introduced the space $R(X)$. We could have done the same for proving Theorem 2.1 introducing the analogous space $R_{b v}(X)$ of sequences in $X$ lying in ranges of measures of bounded variation. In this case we would see that every unconditionally convergent series $\sum x_{n}^{*}$ in $X^{*}$ defines (as in Proposition 3.4(c)) a continuous linear form in $R_{b v}(X)$; so dualizing the condition $c_{0}(X) \subset R_{b v}(X)$ we would obtain that every unconditionally convergent series in $X^{*}$ is absolutely convergent and, by Dvorestky-Rogers Theorem, $X^{*}$ is finite dimensional.

Conversley, the implication (a) $\Rightarrow$ (e) of Theorem 3.6 could be proved using the methods intorduced in $\S 2$; we would prove, via an analogous claim to that in Theorem 2.1, that every finite-dimensional quotient of $X$ is $C$-isomorphic to a quotient of $L^{\infty}$ ( $C$ is an absolute constant). Then, every finite-dimensional subspace of $X^{*}$ is $C$-isomorphic to a subspace of $L^{1}$, implying (see [LP]) that $X^{*}$ can be embedded isomorphically in an $L^{1}$ space.

In Theorem 3.6 we proved that for a Banach space the statements "every null sequence lies inside the range of a measure" and "every null sequence lies in a countable sum of segments" are equivalent. The next example shows that this equivalence is not true for isolated null sequences and proves, by Proposition 1.4 , that not every compact subset of the range of a measure is a subset of a compact range.

Example 3.8. There exists a null sequence in $L^{1}[0,1]$ that lies in the range of a measure but not in any countable sum of segments.

Proof. We know that the unit ball of $L^{2}$ is the range of an $L^{2}$-valued measure (see [AD] or [R]), so it is also the range of an $L^{1}$-valued measure. For $n \in \mathbb{N}$ and $1 \leq k \leq n$ let $f_{n, k}$ be

$$
f_{n, k}=\sqrt{n} \chi_{\left[\frac{k-1}{n}, \frac{k}{n}\right]} \cdot
$$

Since $\left\|f_{n, k}\right\|_{L^{2}}=1$ and $\left\|f_{n, k}\right\|_{L^{1}}=1 / \sqrt{n}$, the sequence $\left(f_{n, k}\right)_{n \in \mathbb{N}, 1 \leq k \leq n}$ is a null sequence in $L^{1}$, which is contained in the range of an $L^{1}$-valued measure. 
We are going to prove that this sequence is not contained in any countable sum of segments; in order to do this we need the following lemma.

Lemma 3.9. Let $\sum x_{n}$ be an unconditionally convergent series in $L^{1}$. Then there exists a compact operator $T: l_{2} \rightarrow L^{1}$ such that

$$
\sum_{n=1}^{\infty}\left[-x_{n}, x_{n}\right] \subset T\left(B_{l_{2}}\right) \text {. }
$$

Before proving the lemma we continue the proof of the example. By the lemma it is enough to prove that if $T: l_{2} \rightarrow L^{1}$ is an operator such that $f_{n, k} \in$ $T\left(B_{l_{2}}\right)$ for $n \in \mathbb{N}$ and $k=1, \ldots, n$, then $T$ is not compact; this is done by using volume arguments.

If $T$ were compact, there would be a natural number $N$ such that $T\left(B_{l_{2}}\right)$ could be covered by $N$ balls of radius $\frac{1}{2}$. If $Y_{n}$ is the subspace of $L^{1}$ generated by $\left(f_{n, k}\right)_{k=1}^{n}$, it is easy to see that there is a norm one projection $P_{n}: L^{1} \rightarrow Y_{n}$. So $P_{n} T\left(B_{l_{2}}\right)$ could be covered by $N$ balls of radius $\frac{1}{2}$ centered in points of $Y_{n}$. There is an isometry from $Y_{n}$ onto $l_{1}^{n}$ that maps each $f_{n, k}$ to $e_{k} / \sqrt{n}\left(\left(e_{k}\right)_{k=1}^{n}\right.$ is the natural basis of $\left.l_{1}^{n}\right)$. So, we can suppose that we are in $\mathbb{R}^{n}$ and we can use the $n$-dimensional volume $\operatorname{vol}_{n}$ (the Lebesgue measure in $\mathbb{R}^{n}$ ) to estimate $N$ below. Taking quotient over the kernel of $P_{n} T$, we obtain an $n$-dimensional Hilbert space and thus $P_{n} T\left(B_{l_{2}}\right)=S\left(B_{2}^{n}\right)$ for a certain operator $S: l_{2}^{n} \rightarrow l_{1}^{n}$. If $S\left(B_{2}^{n}\right)$ can be covered by $N$ translates of $\frac{1}{2} B_{1}^{n}$, we have

$$
\operatorname{vol}_{n}\left(S\left(B_{2}^{n}\right)\right) \leq N \operatorname{vol}_{n}\left(\frac{1}{2} B_{1}^{n}\right)=\frac{N}{2^{n}} \operatorname{vol}_{n}\left(B_{1}^{n}\right) .
$$

The following estimates are easy to prove

$$
\operatorname{vol}_{n}\left(B_{1}^{n}\right)=\frac{2^{n}}{n !} \quad \text { and } \quad \operatorname{vol}_{n}\left(B_{2}^{n}\right) \geq \frac{2^{n}}{\sqrt{n !}}
$$

Regarding $S$ as a $n \times n$-matrix, we get from these estimates and (15)

$$
N \geq \frac{2^{n} \operatorname{vol}_{n}\left(S\left(B_{2}^{n}\right)\right)}{\operatorname{vol}_{n}\left(B_{1}^{n}\right)}=\frac{2^{n}|\operatorname{det} S| \operatorname{vol}_{n}\left(B_{2}^{n}\right)}{\operatorname{vol}_{n}\left(B_{1}^{n}\right)} \geq 2^{n} \sqrt{n !}|\operatorname{det} S| .
$$

Since $e_{k} \in S\left(\sqrt{n} B_{2}^{n}\right)$, it follows that $\left\|S^{-1} e_{k}\right\|_{2} \leq \sqrt{n}$ for $k=1, \ldots, n$. Thus the Hilbert-Schmidt norm of $S^{-1}$ is

$$
\left\|S^{-1}\right\|_{H S}=\left(\sum_{k=1}^{n}\left\|S^{-1} e_{k}\right\|_{2}^{2}\right)^{1 / 2} \leq n \text {. }
$$

This implies

$$
\left(\operatorname{det} S^{-1}\right)^{2}=\operatorname{det}\left(\left(S^{-1}\right)^{*} S^{-1}\right) \leq\left(\frac{1}{n} \operatorname{tr}\left(\left(S^{-1}\right)^{*} S^{-1}\right)\right)^{n}=\left(\frac{1}{n}\left\|S^{-1}\right\|_{H S}^{2}\right)^{n} \leq n^{n} .
$$

Using that $e^{n} n ! \geq n^{n}$, this last estimate and (16), we obtain

$$
N \geq 2^{n} \sqrt{n !}|\operatorname{det} S| \geq 2^{n} \sqrt{n !} \frac{1}{\sqrt{n^{n}}} \geq\left(\frac{2}{\sqrt{e}}\right)^{n} .
$$

This leads to a contradiction, since the last inequality should be true for every $n$. 
Proof of Lemma 3.9. We know that there is a compact operator $S: c_{0} \rightarrow L^{1}$ such that

$$
\sum_{n=1}^{\infty}\left[-x_{n}, x_{n}\right]=\overline{S\left(B_{c_{0}}\right)} L^{1}
$$

We are going to prove that $S$ factors compactly through $l_{2}$. For every subset $A \subset \mathbb{N}$ let $P_{A}$ be the natural projection of $c_{0}\left(l_{2}\right)$ onto $l_{\infty}^{A}\left(l_{2}^{A}\right)$, the subspace generated by $\left\{e_{j}: j \in A\right\}$, and let $i_{A}$ denote the natural inclusions. Since $S$ is compact, we can produce a disjoint sequence $\left(A_{n}\right)$ of finite subset of $\mathbb{N}$ such that

$$
\bigcup_{1}^{\infty} A_{n}=\mathbb{N} \text { and }\left\|S i_{A_{n}}\right\| \leq 4^{-n}\|S\| \text { for } n=0,1, \ldots
$$

Being $l_{\infty}^{A_{n}}$ a $C(K)$ space and $L^{1}$ a cotype 2 space, there exists an absolute constant $c$ such thaet $\pi_{2}\left(S i_{A_{n}}\right) \leq c\left\|S i_{A_{n}}\right\| \leq c 4^{-n}\|S\|$ [P, Theorem 5.14]. This implies a factorization through $l_{2}^{A_{n}}$; that is, there exist $\alpha_{n}: l_{\infty}^{A_{n}} \rightarrow l_{2}^{A_{n}}$ and $\beta_{n}: l_{2}^{A_{n}} \rightarrow L^{1}$ such that $S i_{A_{n}}=\beta_{n} \alpha_{n}$ and $\left\|\alpha_{n}\right\|\left\|\beta_{n}\right\| \leq c 4^{-n}\|S\|$. One can arrange it in such a way that $\left\|\alpha_{n}\right\| \leq 2^{-n}$ and $\left\|\beta_{n}\right\| \leq c\|S\| 2^{-n}$. We can now defined two compact operators $\alpha: c_{0} \rightarrow l_{2}$ and $T: l_{2} \rightarrow L^{1}$ as

$$
\alpha=\sum_{n=1}^{\infty} i_{A_{n}} \alpha_{n} P_{A_{n}} \quad \text { and } \quad T=\sum_{n=1}^{\infty} \beta_{n} P_{A_{n}} .
$$

It is easy to see that $S=T \alpha$, and since $\|\alpha\| \leq 1$ we have

$$
\overline{S\left(B_{c_{0}}\right)}=\overline{T \circ \alpha\left(B_{c_{0}}\right)} \subset \overline{T\left(B_{l_{2}}\right)}=T\left(B_{l_{2}}\right) .
$$

The lemma follows by (17).

Remark. The sequence given in Example 3.8 also works in $L^{p}[0,1], 1<p<2$. It is not possible to construct an example like this in every Banach space failing Theorem 3.6; since, for instance, every measure valued in $l_{p}, 1 \leq p<2$, has relatively compact range.

\section{REFERENCES}

[AD] R. Anantharaman and J. Diestel, Sequences in the range of a vector measure, preprint.

[De] D. W. Dean, The equation $L\left(E, X^{* *}\right)=L(E, X)^{* *}$ and the principle of local reflexity, Proc. Amer. Math. Soc. 40 (1973), 146-148.

[D] J. Diestel, Sequences and series in Banach spaces, Graduate Texts in Math., vol. 92, SpringerVerlag, New York, 1984.

[DU] J. Diestel and J. J. Uhl, Vector measures, Math. Surveys Monogr., vol. 15, Amer. Math. Soc., Providence, RI, 1977.

[KK] I. Kluvanek and G. Knowles, Vector measures and control systems, Math. Stud., vol. 20, North-Holland, 1976.

[J] G. J. O. Jameson, Summing and nuclear norms in Banach space theory, Stud. Texts, vol. 8, London Math. Soc., Cambridge Univ. Press, 1987.

[LP] J. Lindenstrauss and A. Petczynski, Absolutely summing operators in $\mathscr{L}_{p}$-spaces and their applications, Studia Math. 29 (1968), 275-326. 
[P] G. Pisier, Factorization of linear operators and geometry of Banach spaces, Conf. Board Math. Sci. Regional Conf. Ser. Math., vol. 60, Amer. Math. Soc., Providence, RI, 1984.

[R] L. Rodriguez-Piazza, The range of a vector measure determines its total variation, Proc. Amer. Math. Soc. 111 (1991), 205-214.

Departamento de Análisis Matemático, Facultad de Matemáticas, Universidad de Sevilla, Aptdo. 1160, Sevilla, 41080, Spain 